\title{
Saliency detection integrating both background and foreground information
}

\author{
Zhengbing Wang, Guili Xu, Zhengsheng Wang, Chunxing Zhu \\ College of Automation Engineering, Nanjing University of Aeronautics and Astronautics, \\ China
}

\begin{abstract}
In this paper, we propose a novel saliency detection algorithm. The saliency of an image element is defined not only as its contrast to the background but as its similarity to the foreground. First, we extract background seeds as well as their spatial layout information from image boundaries to compute the background-based saliency map. Second, we generate a compact foreground region from the first-stage saliency map to describe the appearance and location of the salient object and calculate the foreground-based saliency map accordingly. We integrate these two saliency maps and further refine the unified one to obtain a more smooth and accurate saliency map. Each component of the presented algorithm is evaluated on the public available datasets and the experimental results also show that the presented algorithm achieves favorable performance compared to the state-of-the-art methods.
\end{abstract}

Keywords:

Saliency detection, Background, Foreground, Saliency map

\section{Introduction}

The biological visual system tends to locate the most attractive regions in a scene rapidly and then primarily processes the information of such areas[1]. In recent years, saliency detection has been further studied to simulate this special characteristic of the human vision system, and numerous computational models have been proposed to detect such attractive regions. As a preprocessing operation, saliency detection can also benefit many other computer vision tasks, such as image compression[2, 3], image categorization[4] and object localization[5]. 


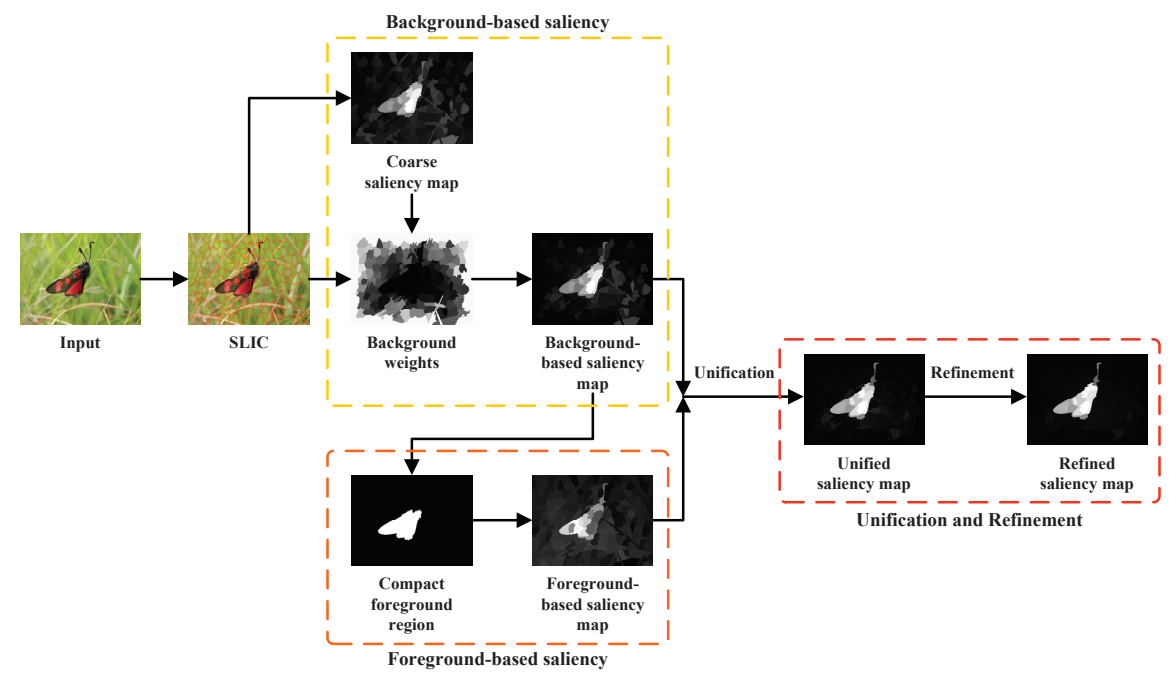

Figure 1: The pipeline of our method, including the background-based saliency, the foreground-based saliency, the unification and refinement operation.

The saliency measure, indicating the dissimilarity of an object to its background, usually forms a saliency map in which each value describes how the pixel or region stands out from the image. From the perspective of information processing, these saliency detection algorithms can be divided into two categories: the bottom-up approaches $[6,7,8,9,10,11,12,13,14,15,16$, $17,18,19]$ and the top-down approaches[20, 21]. The bottom-up approaches are usually data-driven which are concerned with the low-level cues such as color, luminance, texture and location. The top-down approaches are usually task-driven and need to learn the visual information of a specific object to form the saliency map in a supervised manner.

Recent years have witnessed significant progress in visual saliency detection models. The most commonly adopted method is the center-surround contrast which measures the saliency of pixels or regions by the dissimilarity to their surroundings. Itti et al.[6] propose a bottom-up model in which the center-surround operations are implemented on three feature channels in different scales to predict the focus of attention. Harel et al.[7] present a graph-based method and apply random walks to calculate visual saliency. In frequency domain, Hou et al.[10] calculate the spectrum residual (SR) as a indicator of image saliency. More recently, Achanta et al.[9] develop a frequency-turned approach by comparing the color feature of a pixel to the 
average value of the whole image. While this method is simple and efficient, it fails to detect the salient object in complex scene. Cheng et al.[14] propose a global contrast based saliency model which includes both color contrast and spatial coherence. Taking context information into consideration, Goferman et al.[13] develop a new model to detect context-aware saliency which aims at highlighting the image regions that represent the scene. To further improve the performance of saliency model, Jiang et al.[15] segment the input image with a set of parameters and adopt multi-scale contrast to enhance their saliency map. Regarding the image boundary as background region, Wei et al.[16] define the saliency of an image region as its shortest geodesic distance to the virtual background node. Due to the insufficiency of low level features, many algorithms have also incorporated higher level features. Xie et al.[19] propose a bottom-up saliency approach which integrates both low and mid level cue using the Bayesian framework. To detect the salient objects rather than regions, object-level cues[22, 23] are usually incorporated as hints of the foreground. With the popularity of deep learning[24] in many computer vision fields, deep models $[25,26]$ are also introduced in saliency detection, which can achieve favorable performance with proper training strategies.

In this paper, we focus on the bottom-up saliency detection approach and propose a novel saliency detection algorithm based on both background and foreground information. The border regions of the image have been proved to be good visual cues for background priors in saliency detection. Different from previous methods[16, 27] that only compute difference of each image region from the border regions to measure saliency, we further consider the spatial layout of border regions as part of the background information. We generate a compact foreground region from the background-based saliency map to describe the salient object. While other recent works also use foreground information extracted from convex hull[19] or Otsu's threshold method[28] to improve performance of saliency detection, they do not take the compactness of salient object into consideration. The extracted foreground information may therefore include undesirable background noises.

The pipeline of our method is shown in Fig. 1. In the first stage, we select the super-pixels along the image boundaries as background seeds and calculate the weighted color distance between each super-pixel and the selected seeds to obtain a coarse saliency map. To further suppress the background noises, we define the background weight for each super-pixel using the spatial layout information of background seeds. We improve the coarse map with background weights to obtain the background-based saliency map. In 
the second stage, the foreground-based saliency detection is implemented based on the first-stage saliency map. According to the research of visual psychology[1], we generate a compact and coherent foreground region using parametric maxflow to describe the appearance and location of the salient object. Similar to the first-stage saliency detection, the foreground-based saliency map is obtained by the color and spatial similarity to the foreground region. Finally, we integrate the background-based and the foreground-based saliency map according to their roles in saliency detection, and further refine the unified saliency map using the proposed energy function to obtain a smoother final saliency map. The main contribution of this paper are threefold: 1. We devise a new saliency model that measures the saliency with both background and foreground information. 2. By using the spatial layout information of background seeds, we further suppress the noises in cluttered backgrounds. 3. We generate a compact and coherent foreground region based on the background-based saliency map to describe the salient object, which can benefit the saliency detection.

The rest of this paper is organized as follows. Section 2 describes the details of our algorithm. In Section 3, we compare the proposed algorithm with several previous models and analyze the experimental results. We conclude this paper in Section 4.

\section{The proposed approach}

In this section, we present the details of the proposed saliency detection algorithm. In order to preserve the structural information which will benefit subsequent processing, we generate $N$ super-pixels from the input images by the SLIC algorithm[29] and use them as the minimum units. After that, a background-based saliency map is firstly generated using background information. We then segment the foreground region from the first-stage saliency map and define the foreground-based saliency map. Finally, these two saliency maps are integrated and refined to form the final saliency map.

\subsection{Saliency detection with background information}

Inspired by the psychophysical studies[30], the center bias that salien$\mathrm{t}$ objects are more likely to appear at or near the image center has been widely used in the previous works. But these works tend to highlight the center area instead of salient objects. While the salient objects seldom touch the image boundaries, the background areas are usually connected to 


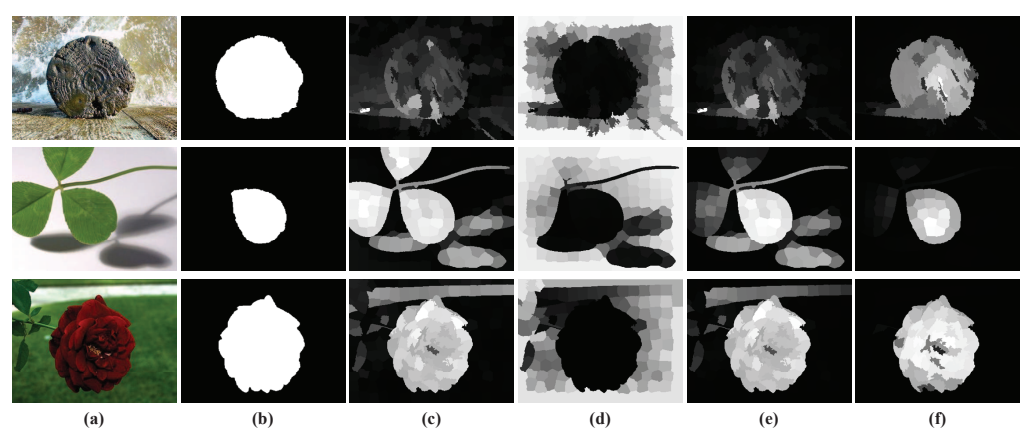

Figure 2: Evaluation of the presented background weights. (a) Input images. (b) Ground truth. (c) Coarse saliency maps. (d) Background weight of each super-pixel. (e) Background-based saliency maps. (f) Final saliency maps.

the boundaries[27]. Therefore, we extract the super-pixels along the image boundaries as the background seeds and define the saliency of super-pixels according to their color contrast to the background ones. A super-pixel is more likely to be part of salient object if it has larger color contrast to the background seeds.

Similar to the previous works, we compute the saliency of a super-pixel as the summation of its color distance to all the background seeds, weighted by their spatial distance. Denote the background seed set as $B G$, and the saliency of super-pixel $s_{i}$ can be calculated as follows:

$$
\widehat{S}_{i}^{b}=\sum_{s_{j} \in B G} d_{c}\left(s_{i}, s_{j}\right) * w_{l}\left(s_{i}, s_{j}\right)
$$

where $d_{c}\left(s_{i}, s_{j}\right)$ is the Euclidean distance of the mean CIELab color vectors of corresponding super-pixels, and $w_{l}\left(s_{i}, s_{j}\right)=\exp \left(-d_{l}^{2}\left(s_{i}, s_{j}\right) / 2 \sigma^{2}\right)$ denotes the spatial weight, in which $d_{l}\left(s_{i}, s_{j}\right)$ is the Euclidean distance between centers of super-pixel $s_{i}$ and $s_{j}$. $\sigma$ represents a constant that controls the strength of the distance.

The illustration of the saliency maps defined as Eq. (1) is shown in Fig. 2(c). We note that these saliency maps are visually unfavorable and some recent works have been presented to improve the basic saliency measurement. In [28], super-pixels with strong edges are regarded as the foreground noises and are roughly removed from background seeds. This operation is simple and efficient. However, as the salient objects seldom touch the image boundaries, it fails to improve the performance in most cases. As shown in Fig. 
2(c), there are usually two shortcomings to measure saliency as Eq. (1). 1. The background seeds cannot highlight the salient object that shares similar features with them. 2. Several background seeds with rare features can be incorrectly highlighted by the other uniform background seeds. Therefore, we further consider the spatial layout of background seeds and define the background weight which denotes the probability of a super-pixel belonging to the background. As the salient objects are spatially compact[21, 31], we define the background weights based on the following heuristic observation. The seeds with widely or discontinuously distributed features are more likely to be background.

To calculate the background weight for each super-pixel, we first cluster the super-pixels in $B G$ into $K$ clusters using the K-means clustering algorith$\mathrm{m}$. The number of clusters $K$ can be chosen according to the complexity of background which is set to 3 in our experiments. For each cluster $k$, find the shortest continuous super-pixel link $S L_{k}$ that contains all the super-pixels belonging to cluster $k$. Denote the length of this super-pixel link as $L_{s}$, and the background weight for each cluster in $B G$ can be calculated as follows:

$$
P_{k}=1-\exp \left(-\alpha\left(L_{s}+L_{o}\right)\right) \quad(k=1,2, \cdots, K)
$$

where $L_{o}$ is the number of super-pixels belonging to the other clusters in $S L_{k}$. $\alpha$ is a constant which is set to 0.05 in our experiments. For each super-pixel $s_{j}$ in cluster $k$, we assign the same value $P_{k}$ to its background weight $p_{s_{j}}$.

The background weights of the remainder super-pixels are determined by their similarity and connectivity to the background seeds in $B G$ :

$$
p_{s_{i}}=\frac{p_{s_{j}^{*}}}{d_{\text {geo }}^{*}} \quad\left(s_{i} \notin B G\right)
$$

where $d_{g e o}^{*}$ is the shortest geodesic distance from super-pixel $s_{i}$ to the background seeds and $s_{j}^{*}$ is the corresponding seed. The main steps of background weight definition are shown in Fig. 3. The background-based saliency is finally calculated as:

$$
S_{i}^{b}=\widehat{S}_{i}^{b} *\left(1-p_{s_{i}}\right)
$$

\subsection{Saliency detection with foreground information}

Referring to [28], the background-based saliency map can highlight all the regions with high contrast and little connectivity to the background seeds. 


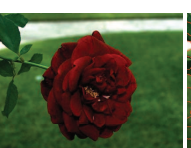

(a)

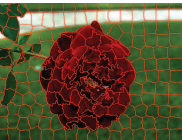

(b)

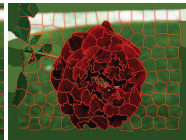

(c)

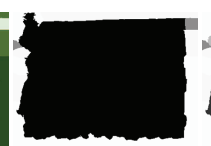

(d)

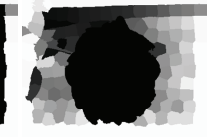

(e)

Figure 3: Main steps of background weight definition. (a) Input images. (b) Super-pixels. (c) Background seeds clustering using k-means. (d) Background weights of the selected background seeds. (e) Background weights of all the super-pixels.

Therefore, it may also include some undesirable background noises as shown in Fig. 4(b). We develop a foreground-based saliency map to suppress these noises based on the description of salient object.

Unlike most previous methods that use local or global contrast to highlight the salient objects. We firstly use background prior to define the background-based saliency map, from which we generate a compact foreground region to describe the salient object and further compute a foregroundbased saliency map. We finally integrate these two maps and thus define the final saliency map with both background and foreground information.

Different from [28] which simply binarizes the saliency map using an adaptive threshold and selects the super-pixels whose background-based saliency values are larger than the threshold as foreground seeds. We take the spatial compactness of salient objects into consideration and use the parametric maxflow[32] to yield the foreground region composed of some spatially coherent super-pixels.

Let $A_{i}$ be the area of super-pixel $s_{i}$. The foreground region can be obtained by solving[33]:

$$
\mathbf{x}^{*}=\min _{\mathbf{x}} \sum_{i=1}^{N}\left(-\ln S_{i}^{b}+\lambda A_{i}\right) x_{i}+\sum_{1<i<j<N} e_{i j} x_{i} x_{j}
$$

where $e_{i j}=\exp \left(-\left(S_{i}^{b}-S_{j}^{b}\right) / 2 \sigma_{s}^{2}\right)$ denotes the similarity between two adjacent super-pixels. $x_{i} \in\{0,1\}$ indicates whether the super-pixel $s_{i}$ belongs to foreground region. Formulation as Eq. (5) not only promotes compactness but also promotes coherence, which is consistent with the characteristic of salient objects. As shown in Fig. 4(d), our approach usually yields more compact solutions than those by adaptive threshold and can more accurately describe the salient objects.

Denote the selected foreground super-pixel set as FG. A super-pixel is more likely to be part of salient object if it is more similar to the fore- 


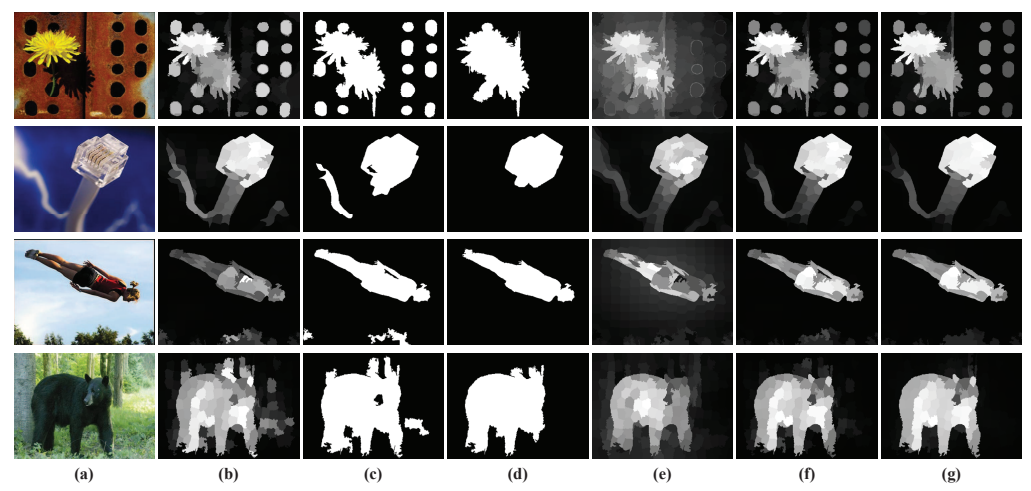

Figure 4: Improvement effects of foreground regions. (a) Input images. (b) Backgroundbased saliency maps. (c) Foreground regions generated by adaptive threshold. (d) Foreground regions generated by the proposed method. (e) Foreground-based saliency maps. (f) Unified saliency maps. (g) Refined saliency maps.

ground region in appearance and spatial location. Similar to the definition of background-based saliency map, we calculate the foreground-based one as the spatial color similarity of each super-pixel with the selected super-pixels in $F G$ :

$$
S_{i}^{f}=\sum_{s_{j} \in F G} \frac{1}{d_{c}\left(s_{i}, s_{j}\right)+\theta d_{l}\left(s_{i}, s_{j}\right)}
$$

where $d_{c}\left(s_{i}, s_{j}\right)$ and $d_{l}\left(s_{i}, s_{j}\right)$ are respectively the Euclidean color and spatial distance between two super-pixels, and parameter $\theta$ balances the importance between color and spatial location distances.

Fig. 4(e) shows some foreground-based saliency maps defined by Eq. (6). The incorrectly highlighted noises in the background-based saliency map are significantly suppressed in the foreground-based one due to the dissimilarity to the foreground region. However, as shown in the third row of Fig. 4, diversified patterns in foreground region sometimes suppress each other. Therefore, we need to integrate the background-based and foreground-based saliency to take their own advantages.

\subsection{Integration and Refinement operation}

The background-based saliency map and the foreground-based saliency map are obtained using background and foreground information respectively in the above subsections. We note that the background-based saliency map 
can uniformly highlight the salient object with some background noises, while the foreground-based one can better suppress the noises. Referring to [28], we integrate them using the following formulation:

$$
S_{i}^{u}=S_{i}^{b} *\left(1-\exp \left(-\beta * S_{i}^{f}\right)\right)
$$

where $\beta$ is the balancing factor between these two saliency maps, which is set to 4 in our experiments. As shown in Fig. 4(f), the unified saliency map can not only highlight the salient object uniformly but also significantly suppress the noises compared with the background-based saliency map.

The spatial color contrast provides a way to highlight the super-pixels with great dissimilarity to background and suppress the ones with great dissimilarity to foreground, but it is insufficient. To obtain a better result, we devise a new energy function to further refine the unified saliency map. According to [34], this energy function should assign large value to foreground area and small value to background area, and it should also promote continuous saliency values. Based on the smoothness property and the unified saliency map, we define the energy function as:

$$
\begin{aligned}
& \mathbf{S}^{r}=\arg \min _{\mathbf{S}}\left(\sum_{i, j=1}^{N} w_{c}\left(s_{i}, s_{j}\right)\left(S_{i}-S_{j}\right)^{2}+\sum_{i=1}^{N} p_{s_{i}}\left(1-\mathbf{x}_{i}^{*}\right)\right. \\
& \left.\left(S_{i}-S_{i}^{u}\right)^{2}+\sum_{i=1}^{N}\left(1-p_{s_{i}}\right) \mathbf{x}_{i}^{*}\left(S_{i}-S_{i}^{u}\right)^{2}\right)
\end{aligned}
$$

where $\mathbf{S}^{r}=\left[S_{1}^{r}, S_{2}^{r}, \cdots, S_{N}^{r}\right]^{T}$ is the refined saliency value vector. There are three terms in the energy function. The first one promotes continuous saliency values within local regions, and $w_{c}\left(s_{i}, s_{j}\right)$ denotes the color similarity between two adjacent super-pixels. In the second term, $\mathbf{x}_{i}^{*}$ denotes the optimal segmentation result of Eq. (5), and this term denotes that the background area with high background weight should take a small value close to its value in the unified saliency map. Similarly, the third term encourages the foreground area to take a large value.

\section{Experiments}

In this section, we evaluate the presented algorithm on three public datasets: MSRA-1000, SED1 and ECSSD. The MSRA-1000 dataset[9] contains 1000 images with pixel-wise labeled ground truth for salient objects. 


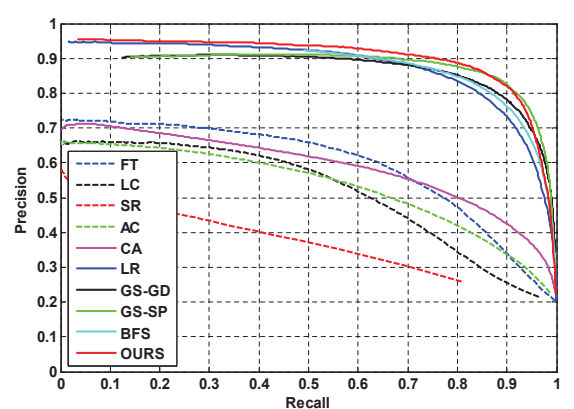

(a)

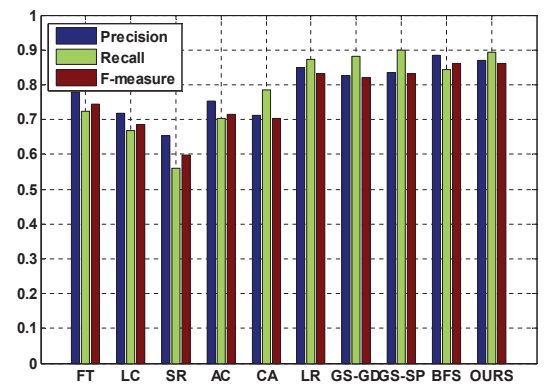

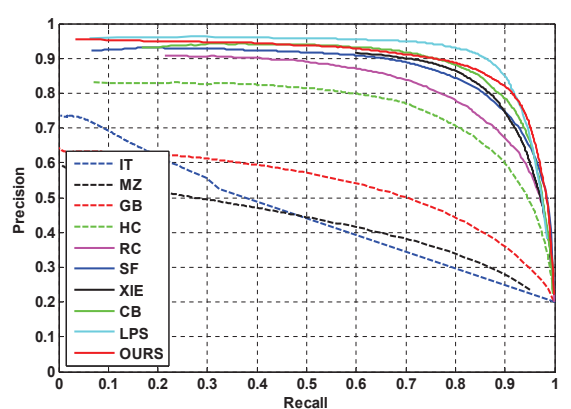

(b)

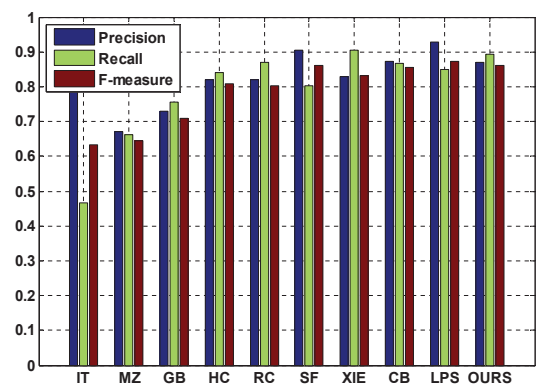

(d)

Figure 5: Quantitative comparison on MSRA-1000 dataset. (a) and (b) The precisionrecall curves of all evaluated models. (c) and (d) The average Precision, Recall and F-measure.

The SED1 dataset[35] includes 100 images with the corresponding accurate masks. The ECSSD dataset[36] consists of 1000 images which generally contain more complex scenes as well as human-labeled masks. In order to evaluate the performance of our algorithm, we compare our algorithm with the previous methods using precision-recall curve and F-measure. Given a saliency map, we segment this map with the threshold ranging from 0 to 255 , and compare each result with the ground truth to generate the precision-recall curve. To calculate the F-measure, we segment the saliency map using an adaptive threshold. The F-measure is the overall performance of precision and recall, and can be obtained by:

$$
F_{\beta}=\frac{\left(1+\beta^{2}\right) \text { Precision } \times \text { Recall }}{\beta^{2} \text { Precision }+ \text { Recall }}
$$

where $\beta^{2}$ is set to 0.3 according to $[9]$. 


\subsection{Comparison with other methods}

We compare our method with 18 state-of-the-art saliency detection models: IT[6], GB[7], MZ[8], FT[9], SR[10], LC[11], AC[12], CA[13], HC[14], RC[14], CB[15], GS-GD[16], GS-SP[16], LR[17], SF[18], XIE[19], BFS[28] and LPS[37] on the MSRA-1000 dataset. To make a fair comparison, we download the saliency maps of IT, GB, MZ, FT, SR, LC, AC, CA, HC and RC from [14]. For GS-GD, GS-SP, SF, XIE and LPS, we use the saliency maps provided by their authors. For CB, LR and BFS, we run the authors' code to obtain saliency maps. The precision-recall curve and F-measure which give a quantitative comparison of these algorithms are shown in Fig. 5. We note that the proposed algorithm achieves good performance against the other state-of-the-art methods. Some visual comparison results are presented in Fig. 6. The IT method can correctly locate the object, but usually highlights parts of the boundary of the salient object. The CA method highlights the object boundary as well as the scene uniformly, but still cannot detect the inner part of the object. Benefit from other computer vision task such as image segmentation, the CB method can more uniformly highlight the salient object. But it may also identify some background patches incorrectly, when these patches are distinct from others. The LR method assumes that the salient object is sparse in the image, which also holds for some distinctive background patches. Therefore, it may emphasize some sparse background patches instead of the salient object. Both the GS and XIE methods can highlight the salient object consistently, but they fail to suppress the background noises. The BFS method can well suppress the noises. However, the salient object may also be suppressed by incorrect foreground seeds. The LPS method is based on label propagation. It can achieve good performance, but may be invalid when it powerlessly segments the salient object from the complex background. Nevertheless, the presented algorithm can uniformly highlight the salient object and restrain the background noises, which therefore provides a desirable saliency detection.

We compare our method with 10 state-of-the-art saliency detection models: IT[6], GB[7], FT[9], SR[10], CB[15], LR[17], XIE[19], SF[18], BFS[28] and LPS[37] on both the SED1 and ECSSD datasets. For each model, we obtain its saliency maps by running the code from the author. The precisionrecall curve and F-measure on the SED1 are shown in Fig. 7, which indicates the SED1 dataset is more challenging than MSRA-1000. In general, the ECSSD dataset is the most challenging among the used three datasets as many images contain more complex scenes. The quantitative comparison results 


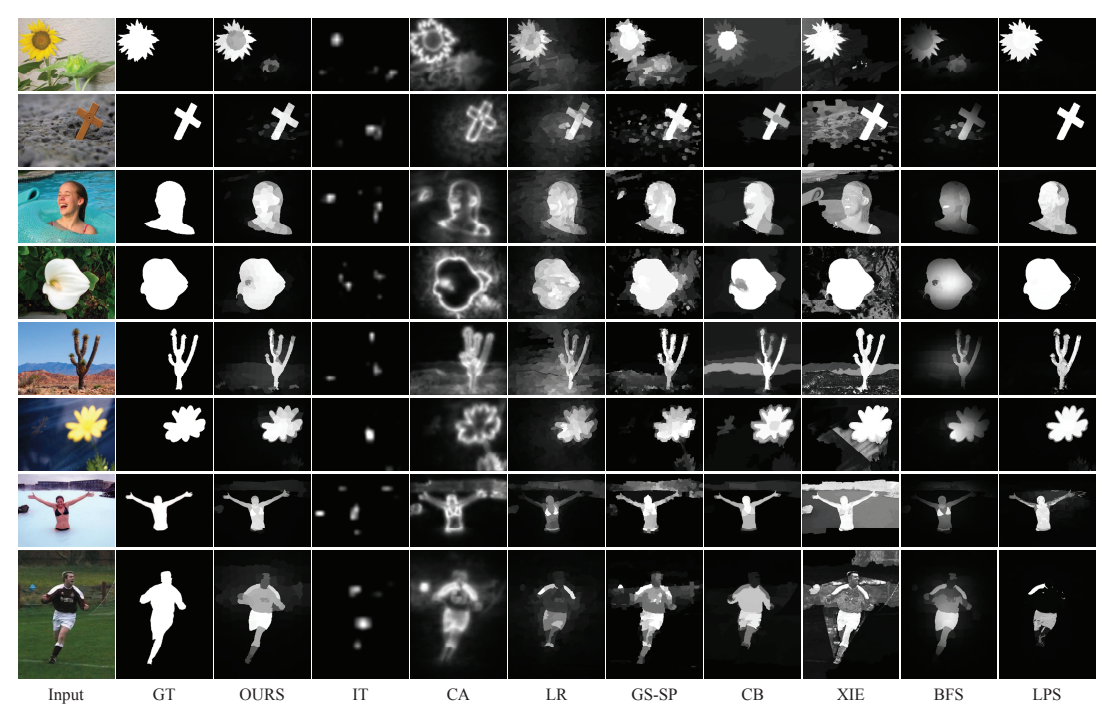

Figure 6: Some visual comparison results of different models on MSRA-1000 dataset.

on the ECSSD is shown in Fig. 8. Some visual comparison results on these two datasets are presented in Fig. 9.

From the quantitative comparison on three public datasets, we note that the proposed algorithm outperforms most of the state-of-the-art method$\mathrm{s}$, including the top-performance models in recent years such as GS and XIE. Compared with GS method which also exploits image boundaries as background prior, our approach can better suppress undesirable noises via foreground information. We generate the compact foreground region based

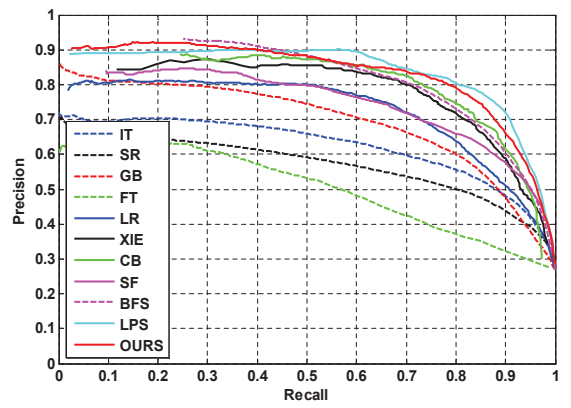

(a)

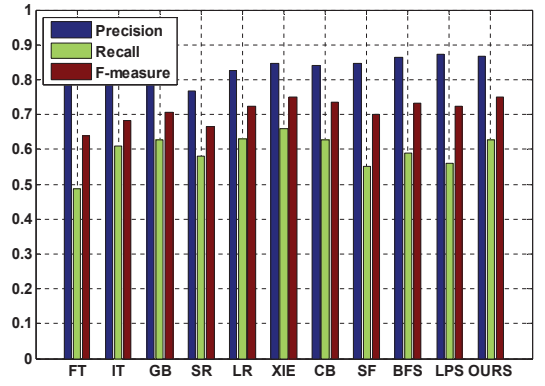

(b)

Figure 7: Quantitative comparison on SED1 dataset. (a) The precision-recall curves of all evaluated models. (b) The average Precision, Recall and F-measure. 


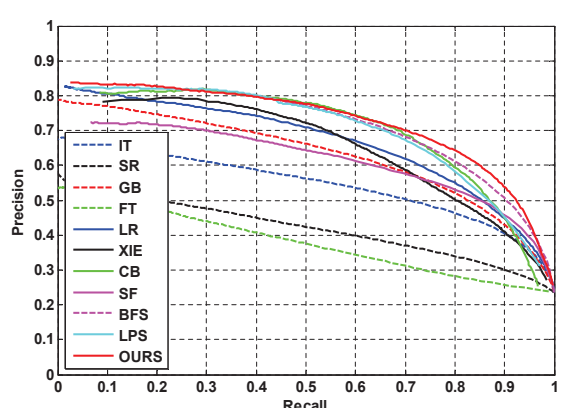

(a)

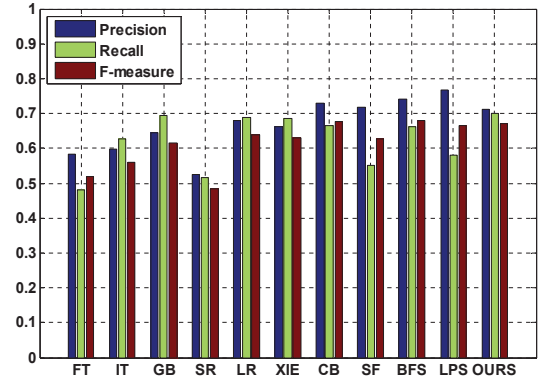

(b)

Figure 8: Quantitative comparison on ECSSD dataset. (a) The precision-recall curves of all evaluated models. (b) The average Precision, Recall and F-measure.

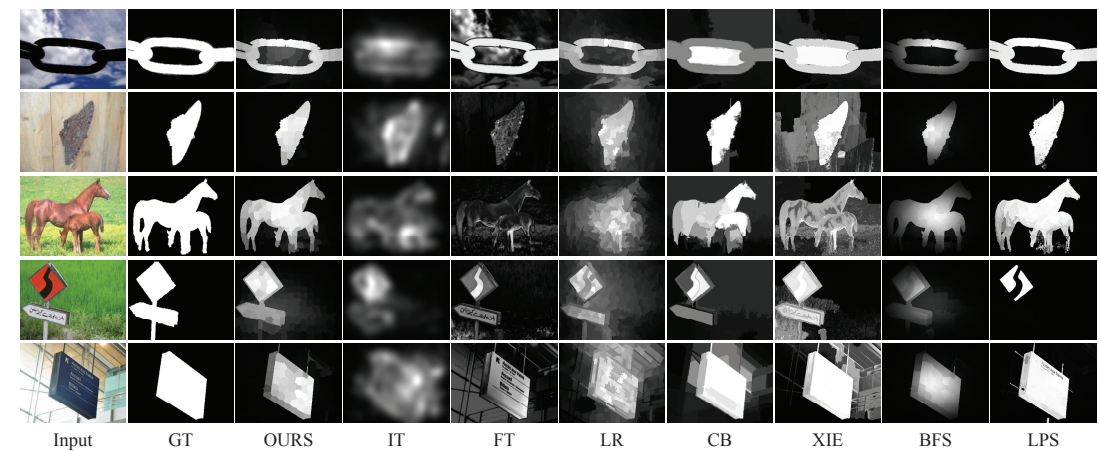

Figure 9: Some visual comparison results of different models on SED1 dataset and ECSSD dataset. 
on super-pixels which retains the contour information, it therefore provides a better description of salient object than convex hull used in XIE method. The effectiveness of our approach can also be demonstrated in visual comparison results.

\subsection{Evaluation of background-based saliency and foreground-based saliency}

As discussed in section 2, we define the coarse saliency of each superpixel as its dissimilarity to background seeds and further improve the coarse saliency map using background weights of super-pixels. To demonstrate the effectiveness of background weights, we compute the precision-recall curves for coarse saliency maps and background-based saliency maps on three public datasets. As shown in Fig. 10, the background-based saliency detection achieves a higher performance than the coarse one, which indicates the presented background weights can substantially improve the coarse saliency maps. Fig. 2(c) and 2(e) show the visual improvement by using background weights. To evaluate the compact foreground region generated from the background-based saliency map, we also compute the precision-recall curves for foreground-based saliency maps. Note that, the foreground-based saliency detection has a lower performance than the background-based one. The main reasons are as follows: 1 . There may be some undesired information in the foreground region which has unfavorable influence on saliency detection as shown in Fig. 11. 2. Diversified patterns in foreground region may mutually suppress the saliency. However, the foreground-based result also contributes to the unified result in Fig. 10, as the background-based result and the foreground-based one complement each other in saliency detection. Fig. 4(e) shows the foreground-based saliency maps suppress background noises more than the background-based ones.

\subsection{Evaluation of the refinement operation}

We refine the unified saliency map by the energy function presented in subsection 2.3. As shown in Fig. 4(g), the proposed refinement operation promotes local smoothness in saliency maps. The refined saliency maps can therefore highlight salient objects more uniformly and better suppress background noises. To make a quantitative evaluation, we compute the precisionrecall curves for unified saliency maps and refined saliency maps. The result is shown in Fig. 10 which indicates the final saliency maps refined by the energy function can achieve a better performance. 


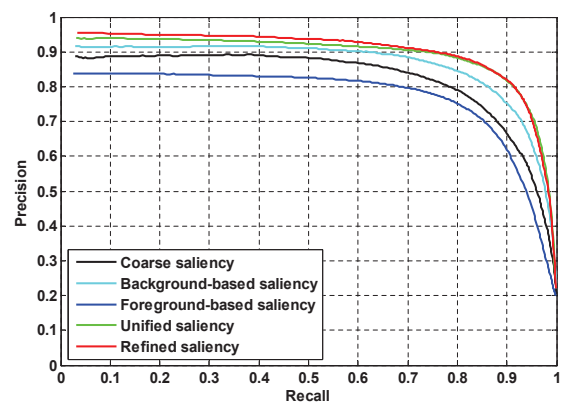

(a)

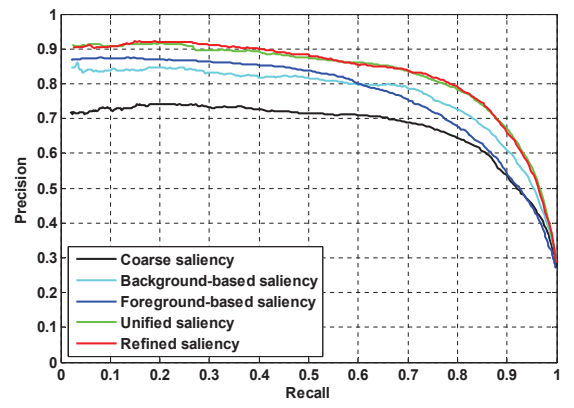

(b)

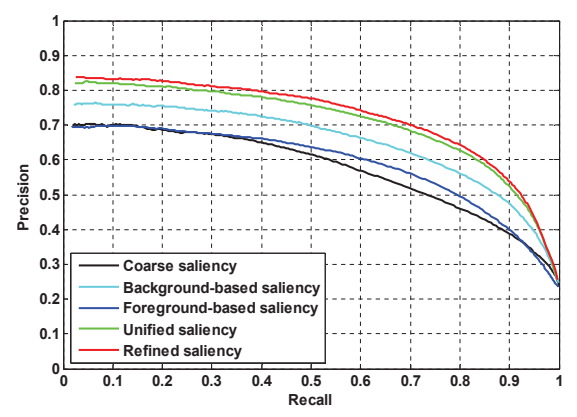

(c)

Figure 10: Evaluation of each component of the presented algorithm on (a) MSRA-1000 dataset, (b) SED1 dataset and (c) ECSSD dataset.

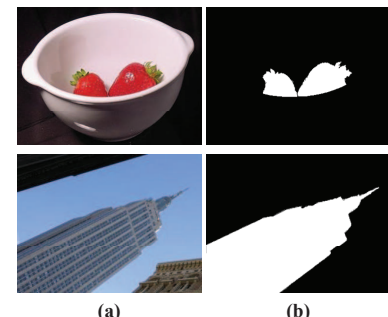

(a)

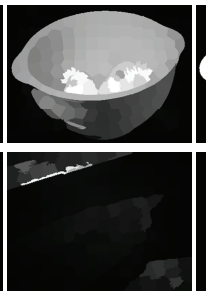

(c)

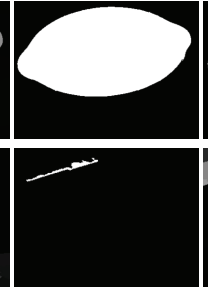

(d)

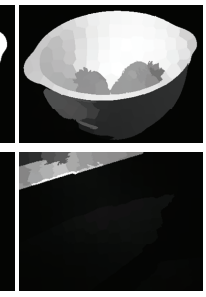

(e)

Figure 11: Failure cases of the proposed algorithm. (a) Input images. (b) Ground truth. (c) Background-based saliency maps. (d) Obtained foreground regions. (e) Final saliency maps. 
Note that, we generate the foreground region from the background-based saliency map. If the saliency map obtained in the first stage is not favorable enough, the foreground region may include some invalid information which may have negative effect on saliency detection instead. Fig. 11 shows some failure cases of the proposed method.

\section{Conclusion}

This paper presents a saliency detection algorithm integrating both background and foreground information which complement each other in saliency detection. We also propose a new energy function which can further refine the unified saliency map. We evaluate the presented algorithm on three public available datasets and the experimental results show that our method achieves favorable performance compared to the state-of-the-art methods.

\section{Acknowledgements}

This project is supported by the National Science Foundation of China (61473148) and the Priority Academic Program Development of Jiangsu Higher Education Institutions.

\section{References}

[1] F. T. Qiu, T. Sugihara, R. von der Heydt, Figure-ground mechanisms provide structure for selective attention, Nature neuroscience 10 (11) (2007) 1492-1499.

[2] C. Guo, L. Zhang, A novel multiresolution spatiotemporal saliency detection model and its applications in image and video compression, IEEE Trans. Image Processing 19 (1) (2010) 185-198.

[3] L. Itti, Automatic foveation for video compression using a neurobiological model of visual attention, IEEE Trans. Image Processing 13 (10) (2004) 1304-1318.

[4] C. Siagian, L. Itti, Rapid biologically-inspired scene classification using features shared with visual attention, IEEE Trans. Pattern Anal. Mach. Intell 29 (2) (2007) 300-312. 
[5] D. Gao, S. Han, N. Vasconcelos, Discriminant saliency, the detection of suspicious coincidences, and applications to visual recognition, IEEE Trans. Pattern Anal. Mach. Intell 31 (6) (2009) 989-1005.

[6] L. Itti, C. Koch, E. Niebur, A model of saliency-based visual attention for rapid scene analysis, IEEE Trans. Pattern Anal. Mach. Intell 20 (11) (1998) 1254-1259.

[7] J. Harel, C. Koch, P. Perona, Graph-based visual saliency, in: Advances in neural information processing systems, 2006, pp. 545-552.

[8] Y. Ma, H. Zhang, Contrast-based image attention analysis by using fuzzy growing, in: Proceedings of the eleventh ACM international conference on Multimedia, ACM, 2003, pp. 374-381.

[9] R. Achanta, S. Hemami, F. Estrada, S. Susstrunk, Frequency-tuned salient region detection, in: Proceedings of IEEE Conference on Computer Vision and Pattern Recognition, IEEE, 2009, pp. 1597-1604.

[10] X. Hou, L. Zhang, Saliency detection: A spectral residual approach, in: Proceedings of IEEE Conference on Computer Vision and Pattern Recognition, IEEE, 2007, pp. 1-8.

[11] Y. Zhai, M. Shah, Visual attention detection in video sequences using spatiotemporal cues, in: Proceedings of the 14th annual ACM international conference on Multimedia, ACM, 2006, pp. 815-824.

[12] R. Achanta, F. Estrada, P. Wils, S. Süsstrunk, Salient region detection and segmentation, in: Computer Vision Systems, Springer, 2008, pp. $66-75$.

[13] S. Goferman, L. Zelnik-Manor, A. Tal, Context-aware saliency detection, IEEE Trans. Pattern Anal. Mach. Intell 34 (10) (2012) 1915-1926.

[14] M. Cheng, N. J. Mitra, X. Huang, P. H. Torr, S. Hu, Global contrast based salient region detection, IEEE Trans. Pattern Anal. Mach. Intell 37 (3) (2015) 569-582.

[15] H. Jiang, J. Wang, Z. Yuan, T. Liu, N. Zheng, S. Li, Automatic salient object segmentation based on context and shape prior., in: Proceedings of British Machine Vision Conference, 2011, pp. 1-12. 
[16] Y. Wei, F. Wen, W. Zhu, J. Sun, Geodesic saliency using background priors, in: Proceedings of European Conference on Computer Vision, Springer, 2012, pp. 29-42.

[17] X. Shen, Y. Wu, A unified approach to salient object detection via low rank matrix recovery, in: Proceedings of IEEE Conference on Computer Vision and Pattern Recognition, IEEE, 2012, pp. 853-860.

[18] F. Perazzi, P. Krähenbühl, Y. Pritch, A. Hornung, Saliency filters: Contrast based filtering for salient region detection, in: Proceedings of IEEE Conference on Computer Vision and Pattern Recognition, IEEE, 2012, pp. 733-740.

[19] Y. Xie, H. Lu, M.-H. Yang, Bayesian saliency via low and mid level cues, IEEE Trans. Image Processing 22 (5) (2013) 1689-1698.

[20] L. Zhang, M. H. Tong, T. K. Marks, H. Shan, G. W. Cottrell, Sun: A bayesian framework for saliency using natural statistics, Journal of vision 8 (7) (2008) 1-20.

[21] T. Liu, Z. Yuan, J. Sun, J. Wang, N. Zheng, X. Tang, H.-Y. Shum, Learning to detect a salient object, IEEE Trans. Pattern Anal. Mach. Intell 33 (2) (2011) 353-367.

[22] G. Zhong, R. Liu, J. Cao, Z. Su, A generalized nonlocal mean framework with object-level cues for saliency detection, The Visual Computer (2015) 1-13.

[23] C. Jia, J. Qi, X. Li, H. Lu, Saliency detection via a unified generative and discriminative model, Neurocomputing 173 (2016) 406-417.

[24] Y. Guo, Y. Liu, A. Oerlemans, S. Lao, S. Wu, M. S. Lew, Deep learning for visual understanding: A review, Neurocomputing 187 (2016) 27-48.

[25] L. Wang, H. Lu, X. Ruan, M.-H. Yang, Deep networks for saliency detection via local estimation and global search, in: Proceedings of IEEE Conference on Computer Vision and Pattern Recognition, IEEE, 2015, pp. 3183-3192.

[26] R. Zhao, W. Ouyang, H. Li, X. Wang, Saliency detection by multicontext deep learning, in: Proceedings of IEEE Conference on Computer Vision and Pattern Recognition, IEEE, 2015, pp. 1265-1274. 
[27] M. Xu, H. Zhang, Saliency detection with color contrast based on boundary information and neighbors, The Visual Computer 31 (3) (2015) 355364.

[28] J. Wang, H. Lu, X. Li, N. Tong, W. Liu, Saliency detection via background and foreground seed selection, Neurocomputing 152 (2015) 359368.

[29] R. Achanta, A. Shaji, K. Smith, A. Lucchi, P. Fua, S. Susstrunk, Slic superpixels compared to state-of-the-art superpixel methods, IEEE Trans. Pattern Anal. Mach. Intell 34 (11) (2012) 2274-2282.

[30] P. Tseng, R. Carmi, I. G. Cameron, D. P. Munoz, L. Itti, Quantifying center bias of observers in free viewing of dynamic natural scenes, Journal of vision 9 (7) (2009) 74-76.

[31] M. Cheng, J. Warrell, W. Lin, S. Zheng, V. Vineet, N. Crook, Efficient salient region detection with soft image abstraction, in: Proceedings of IEEE International Conference on Computer Vision, IEEE, 2013, pp. $1529-1536$.

[32] A. Levinshtein, C. Sminchisescu, S. Dickinson, Optimal contour closure by superpixel grouping, in: Proceedings of European Conference on Computer Vision, Springer, 2010, pp. 480-493.

[33] V. Kolmogorov, Y. Boykov, C. Rother, Applications of parametric maxflow in computer vision, in: Proceedings of IEEE International Conference on Computer Vision, IEEE, 2007, pp. 1-8.

[34] W. Zhu, S. Liang, Y. Wei, J. Sun, Saliency optimization from robust background detection, in: Proceedings of IEEE Conference on Computer Vision and Pattern Recognition, IEEE, 2014, pp. 2814-2821.

[35] S. Alpert, M. Galun, A. Brandt, R. Basri, Image segmentation by probabilistic bottom-up aggregation and cue integration, IEEE Trans. Pattern Anal. Mach. Intell 34 (2) (2012) 315-327.

[36] Q. Yan, L. Xu, J. Shi, J. Jia, Hierarchical saliency detection, in: Proceedings of IEEE Conference on Computer Vision and Pattern Recognition, IEEE, 2013, pp. 1155-1162. 
[37] H. Li, H. Lu, Z. Lin, X. Shen, B. Price, Inner and inter label propagation: Salient object detection in the wild, IEEE Trans. Image Processing 24 (10) (2015) 3176-3186. 
Zhengbing Wang received the M.S. degree in control theory and control engineering from Northeastern University, Shenyang, China, in 2014. His current research interests include saliency detection and feature extraction.

Guili Xu is a professor in the College of Automation Engineering at Nanjing University of Aeronautics and Astronautics, Nanjing, China. He received his Ph.D. degree from Jiangsu University in 2002. His current research interests are computer vision and intelligent system.

Zhengsheng Wang is a professor in the College of Science at Nanjing University of Aeronautics and Astronautics, Nanjing, China. He received his Ph.D. degree from Nanjing University of Aeronautics and Astronautics in 2006. His current research interests are computational mathematics, matrix theory computation and applications, and matrix methods in data mining and pattern recognition.

Chunxing Zhu is a graduate student in the College of Automation Engineering at Nanjing University of Aeronautics and Astronautics, Nanjing, China. His current research interests are computer vision and intelligent system. 
${ }^{*}$ Photo of the author(s)

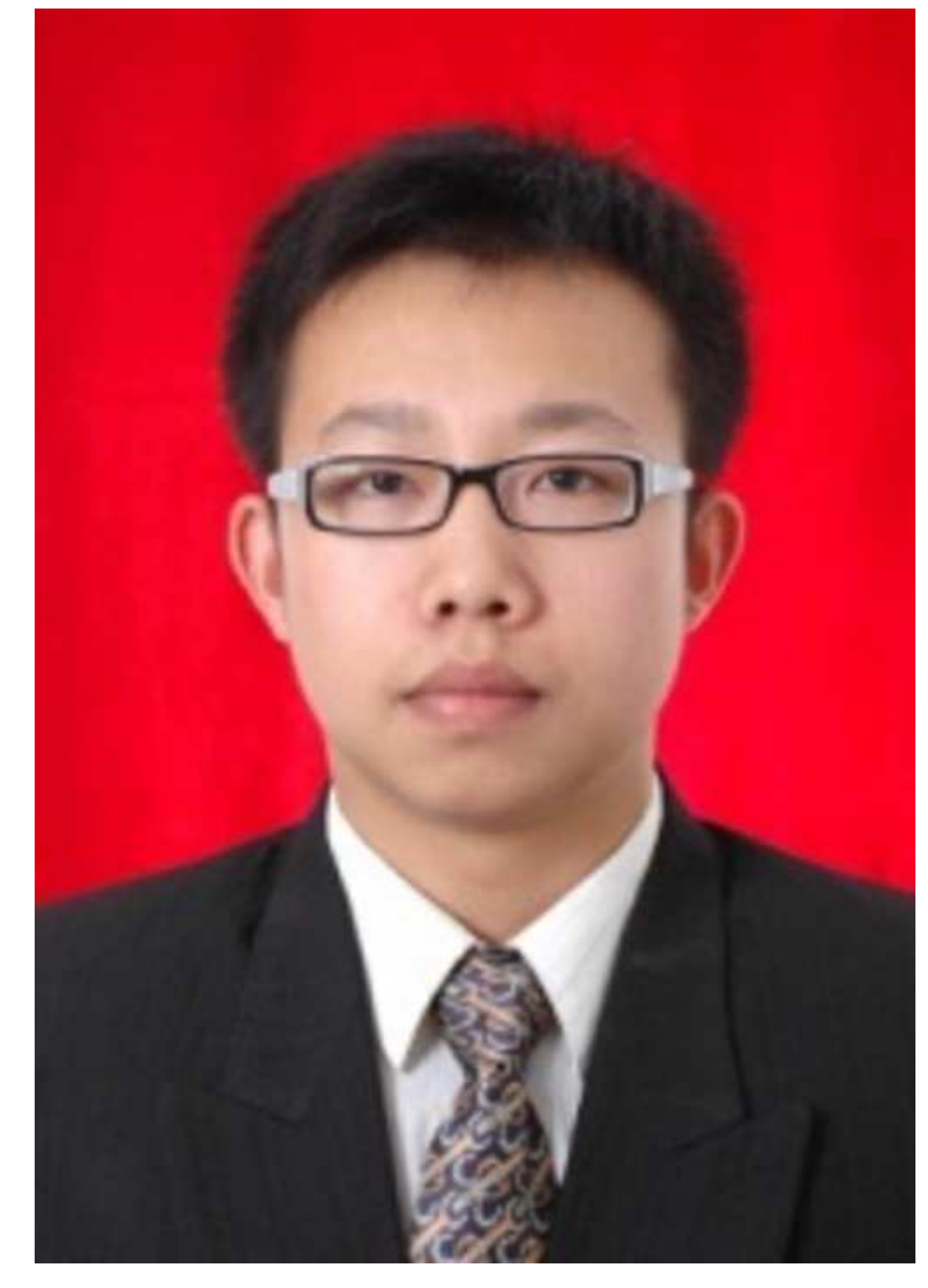




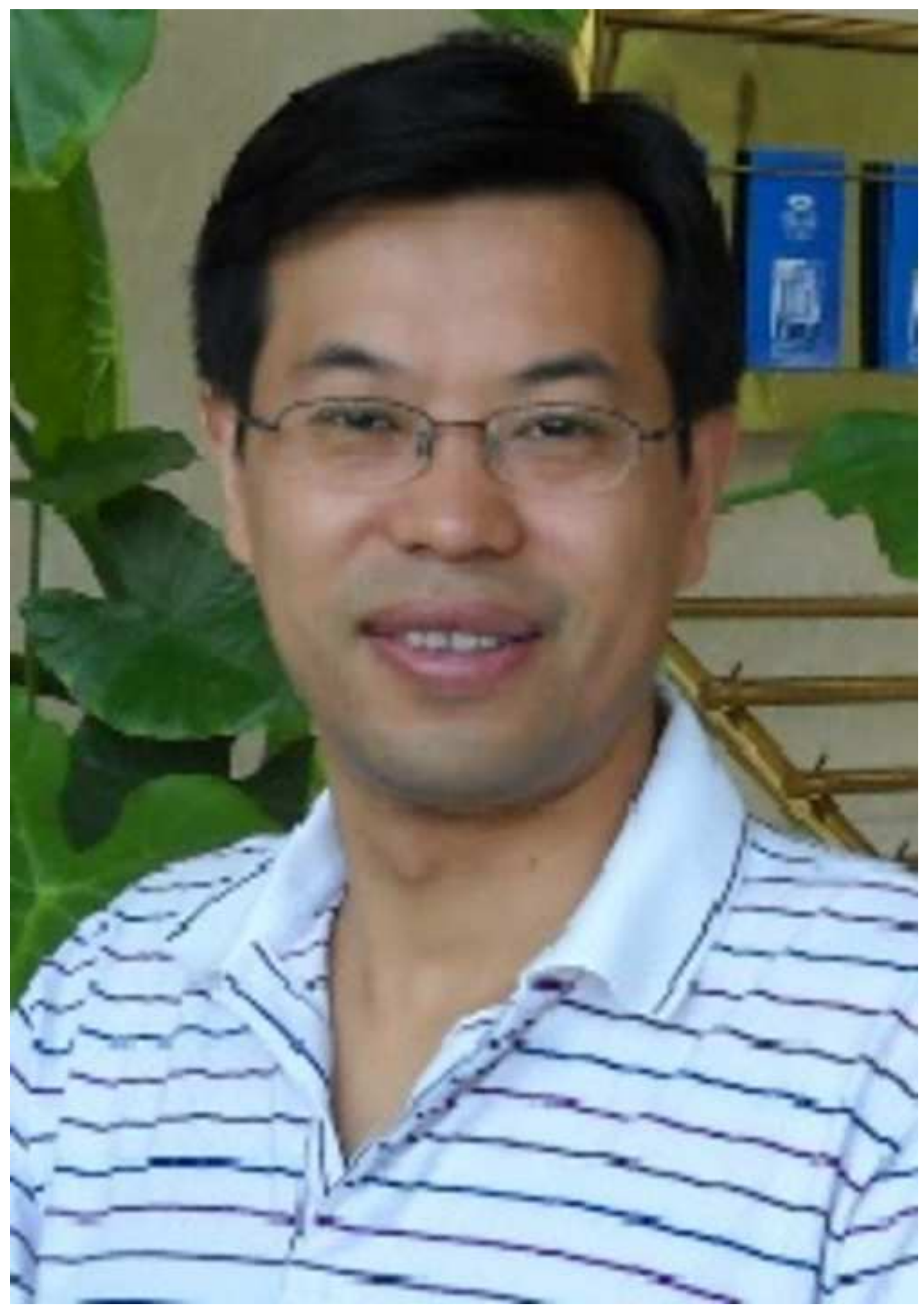




\section{${ }^{*}$ Photo of the author(s)}
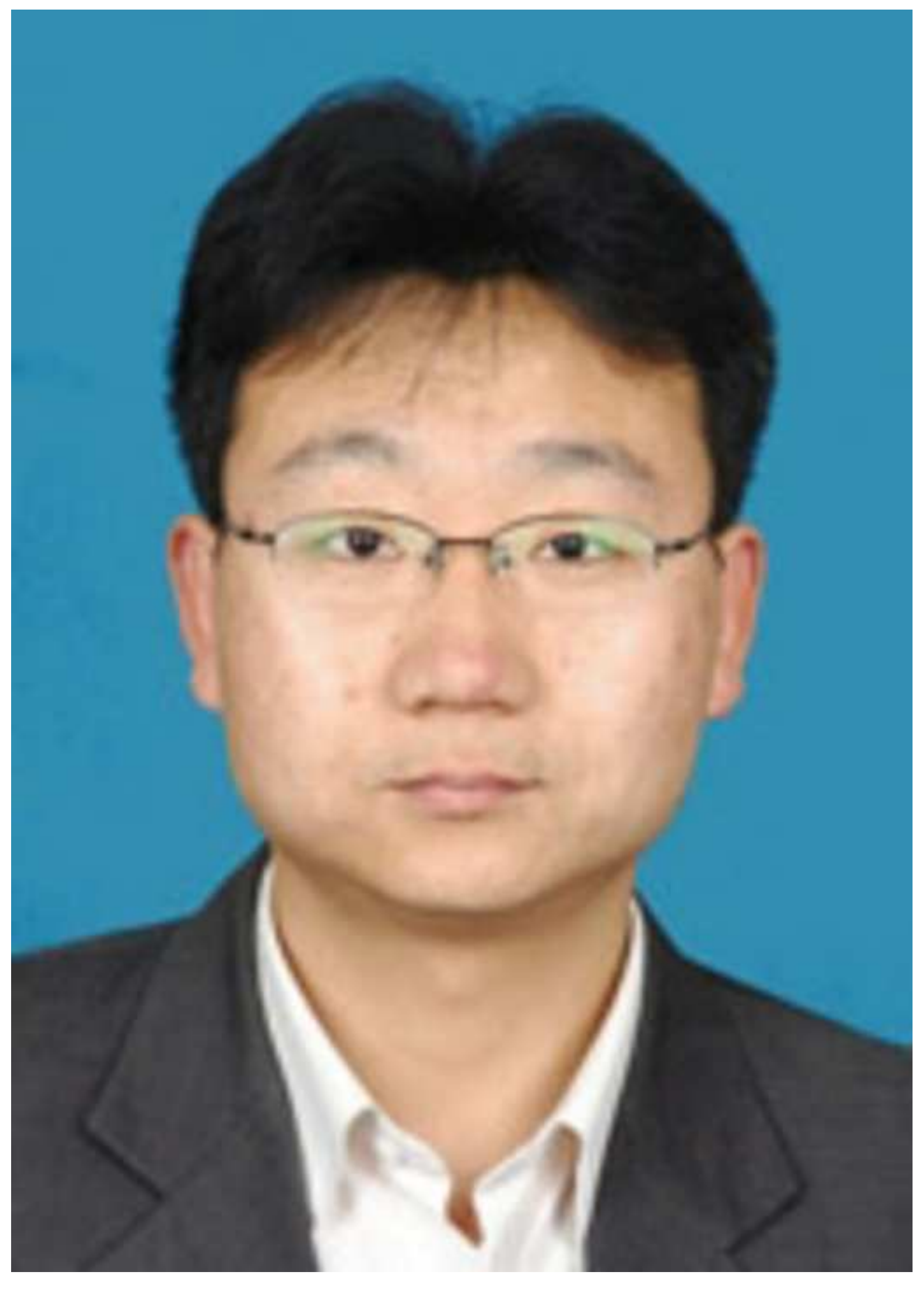
${ }^{*}$ Photo of the author(s)

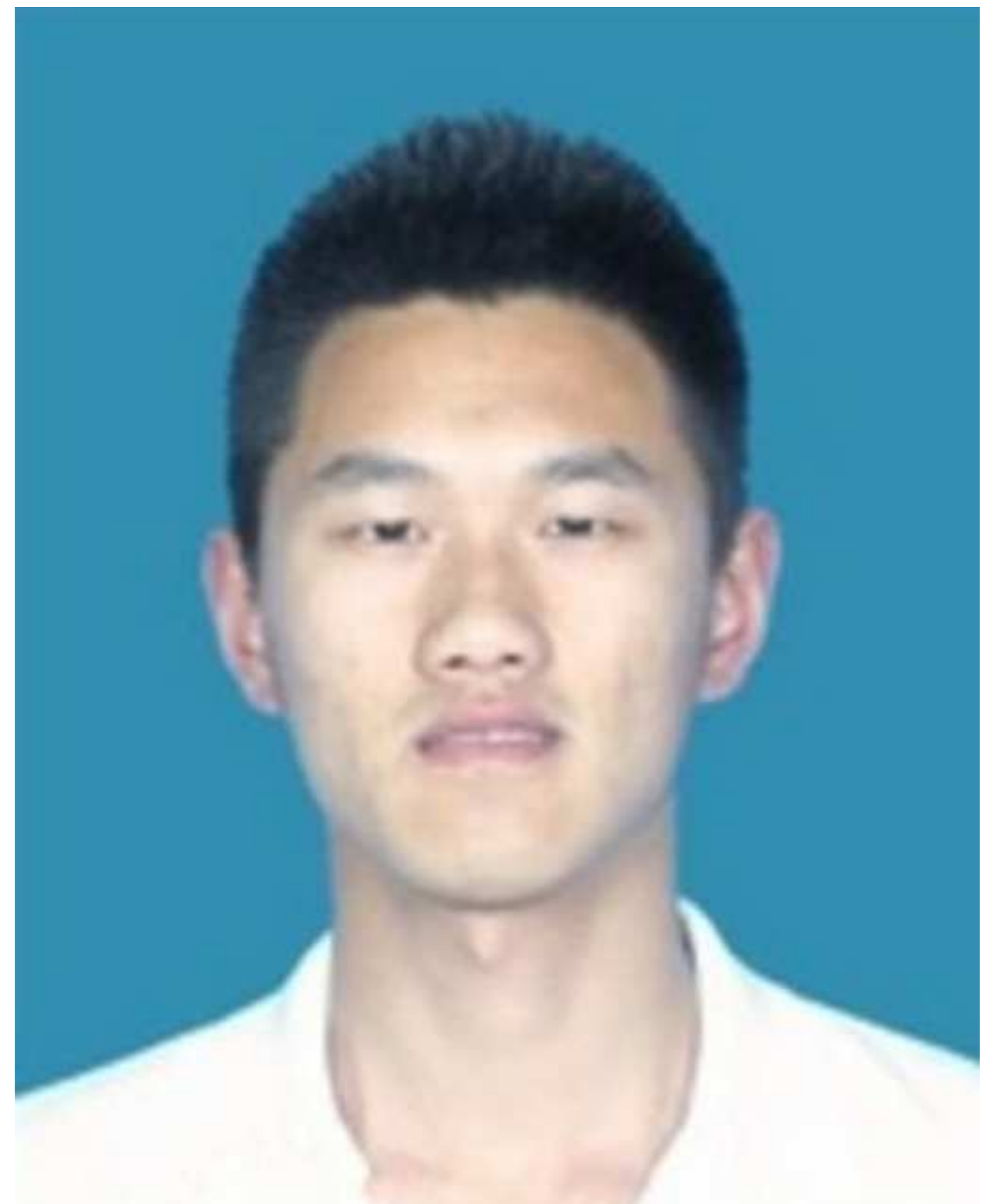

